\title{
METODOLOGÍA BASADA EN EL MODELO SCOR PARA ANALIZAR EL PROCESO DE PRODUCCIÓN DE ABONO ORGÁNICO EN LOMBRICULTIVOS
}

\section{METHODOLOGY BASED ON THE SCOR MODEL TO ANALYZE THE PROCESS OF PRODUCTION OF ORGANIC FERTILIZER IN LOMBRICULTIVOS}

\author{
Ing. Karla Yohana Sánchez-Mojica*, Ph.D Luis Asunción Pérez \\ Dominguez **, Ph.D Miguel Rojas Santiago ***, Ing. Kevin Rafael Palomino \\ Pacheco ${ }^{* * *}$
}

${ }^{*}$ Fundación de Estudios Superiores Comfanorte FESC, Unidad de Investigaciones, Grupo de Investigación GRINFESC. investigaciones@fesc.edu.co, Cúcuta, Norte de Santander, Colombia. 3154324250

** Universidad Autónoma de Ciudad Juárez, Facultad de Ingenierías, Grupo de Investigación GRINFESC. luis.dominguez@uacj.mx, Ciudad Juárez, México. $+5216565832776$

**:* Universidad del Norte, Departamento de Ingeniería Industrial, Grupo de Investigación Productividad y Competitividad.

(Miguelrojas, krpalomino)@uninorte.edu.co, Barranquilla, Departamento del Atlántico, Colombia.

57-5-3509509 ext. 3816

Resumen: En este documento se presenta un análisis para la producción de abono orgánico en lombricultivos usando el Modelo de Referencia para Cadenas de Suministro - SCOR. SCOR es un modelo estándar basado en una estructura que permite eslabonar procesos logísticos, procesos de reingeniería, indicadores de desempeño, benchmarking, mejores prácticas y tecnologías dentro de la cadena de suministro. Además, por medio del modelo SCOR se pueden encontrar los componentes de la cadena para la generación de una SCORCARD que ayude en la administración. En la cadena de suministro de generación de abono orgánico a partir de la lombriz roja californiana, se identifican los niveles del proceso, indicadores clave de desempeño y atributos a modo de diagnóstico para posteriormente sugerir una SCORCARD ajustada al contexto. En este sentido, se pudo evidenciar que la aplicación del modelo SCOR organiza y permite mejores mediciones a lo largo de la producción.

Palabras Clave: abono, lombricultivo, orgánico, producción, SCOR.

\begin{abstract}
This document presents an analysis for the production of organic fertilizer in vermiculture crops using the Reference Model for Supply Chains - SCOR. SCOR is a standard model based on a structure that allows linking logistics processes, reengineering processes, performance indicators, benchmarking, best practices and technologies within the supply chain. Thus, through the SCOR model can be find the components of the chain for the generation of a SCORCARD that helps in the administration. In the supply chain for the generation of organic fertilizer from the California redworm, the process levels, key performance indicators and attributes are identified by way of diagnosis to later suggest a context-adjusted SCORCARD. In this sense, it was evident that the application of the SCOR model organizes and allows better measurements throughout the production.
\end{abstract}

Keywords: fertilizer, vermiculture, organic, production, SCOR 


\section{INTRODUCCIÓN}

La administración de las cadenas de suministro es un problema complejo que durante años se viene estudiando con el fin de entregar a los gerentes nuevas herramientas que les ayuden en su gestión (Santiago, 2006). En las pequeñas y medianas empresas se hace más difícil la administración de las cadenas de suministro debido a los escasos recursos (Santos, 2019), por lo que, no son suficientes para adquirir nuevas tecnologías o mecanismos que faciliten su gestión.

Por otro lado, mantenerse en el mercado y ser más competitivas es el ideal de las empresas, sin embargo, muchas veces se presenta obstáculos de carácter interno que impiden hacer seguimiento a los indicadores que se miden a lo largo de la cadena de suministro y que permiten la mejora continua. Con los grandes retos de un mercado globalizado, las cadenas de suministro deben mejorar aspectos como la coordinación e integración de procesos para facilitar el análisis y control del cumplimiento de los objetivos, así como facilitar la toma de decisiones (Curbelo \& Delgado, 2014).

El modelo SCOR (Modelo Supply Chain Operations Reference) es una metodología validada en aplicaciones a procesos del sector productivo (Naranjo, Reyes, \& Rodríguez, 2012). De este modo, el SCOR es definido por el Supply Chain Council (SCC) como un modelo estándar basado en una estructura que permite eslabonar procesos logísticos, procesos de reingeniería, indicadores de desempeño, benchmarking, mejores prácticas y tecnologías dentro de la cadena de suministro, lo cual debe permitir mejorar su gestión y la relación entre sus actores (Supply Chain Council, 2008). En este sentido, el modelo permite estandarizar el lenguaje para la compresión del funcionamiento de la cadena de suministro y permite tener una visión del sistema en su totalidad. Está compuesto por indicadores que buscan medir su comportamiento a nivel interno (costos y activos) y externos (fiabilidad, respuesta y flexibilidad) de forma general, facilitando la toma de decisiones (Mazo, Montoya, \& Henao, 2014).

Igualmente, en una revisión de casos de aplicación del modelo SCOR en la industria, realizada por Delipinar \& Kocaoglu (2016), se muestra que sus beneficios no han sido lo suficientemente explotados. Además, la revisión realizada muestra que 4 artículos son el ejemplo de uso exitoso del modelo SCOR en estudios de investigación, 2 de ellos son sobre alineación estratégica entre negocios y estrategias de Tecnologías de la Información (TI), 3 de ellos son las limitaciones del modelo SCOR y las medidas de rendimiento, 5 son modelos para la gestión de la cadena de suministro y 3 son marcos teóricos.

Así mismo, dentro de la literatura no se encuentra información relacionada con la utilización del modelo SCOR en procesos pertenecientes con la fabricación de abono orgánico ni con su utilización en el diagnóstico de cadenas de suministro en líneas de producción con procesos de exportación ya certificados. Se encuentran casos de su aplicación en diferentes áreas o sectores que están directamente relacionadas con procesos de manufactura y con frecuencia para diagnósticos empresariales (Dissanayake \& Cross, 2018).

De esta manera, para desarrollar el diagnóstico del comportamiento logístico de la cadena de suministro de producción de abono a base de la lombriz roja californiana en el departamento Norte de Santander (Colombia), objeto de estudio de la presente investigación, se utiliza la metodología del Modelo SCOR, teniendo en cuenta que es un modelo de referencia que estandariza la terminología y los procesos de una cadena de suministro para modelar y, usando KPI (Key Performance Indicators o Indicadores Claves de Desempeño), comparar y analizar diferentes alternativas y estrategias de la cadena de suministro (Naranjo, Reyes, \& Rodríguez, 2012). De tal suerte, aunque los indicadores (KPI) que propone SCOR apoyan la medición de los diferentes procesos de la cadena suministro se vuelve complicado muchas veces adaptarlos a los contextos (Akkawuttiwanich \& Yenradee, 2018). Por esta razón, en el caso de estudio acerca de la industria productora de abono orgánico, se analizará y se verificarán los KPI desde el punto de vista de una línea de producción con características de exportación donde el producto ya cuenta con un proceso certificado. Por lo que, al realizar un diagnóstico utilizando el modelo SCOR, generalmente termina con la identificación o desarrollo de una propuesta de SCORCARD, que es la tarjeta de métricas que se establece para un caso de estudio utilizando el modelo SCOR (Curbelo \& Delgado, 2014). Una SCORCARD además de informar, contribuye a formular y a comunicar la estrategia, alinear los objetivos de la organización y de los empleados, formar y motivar 
a los trabajadores, mejorar de manera continua $\mathrm{y}$, por último, rediseñar la estrategias.

Para lograr establecer una SCORCARD por proceso en una empresa es un gran avance en las mediciones para garantizar la competitividad (Lepori, Damand, \& Barth, 2013). Por tal razón, en este estudio se busca establecer una SCORCARD que se ajuste a la cadena de suministro de producción de abono orgánico teniendo en cuenta las necesidades de los clientes, la visión de la empresa y las buenas prácticas.

Por otra parte, el interés por analizar empresas productoras de abono orgánico específicamente con la característica de utilización de la lombriz californiana tiene que ver con las buenas prácticas reportadas por esta industria. La producción de abono orgánico en los últimos años ha mostrado grandes resultados como por ejemplo la conservación del suelo, la reutilización de materias o desechos que antes contaminaban el ambiente, aumento de los nutrientes en el producto, lo que generó una gran explotación de este método (Souza, Carmo, Santos, \& Fernandes, 2018).

El abono orgánico es un producto con protocolos de higiene y estabilización que se genera de la descomposición biológica oxidativa de materiales orgánicos frescos de deshechos animales y vegetales (Penagos \& Rodríguez, 2009). Se obtiene mediante el procedimiento de descomposición de materiales orgánicos, en un ambiente controlado y con ayuda de los microorganismos.

Finalmente, el propósito de esta investigación es diagnosticar la cadena de suministro en el campo de la fabricación de abono orgánico a través de la lombriz californiana y sugerir posibles mejoras a través de la elaboración de una SCORCARD con métricas propuestas en el modelo SCOR. Por medio de los indicadores (KPI) de evaluación que propone el modelo de referencia SCOR (Lama \& Esteban, 2005) se analiza la mejor estrategia para avanzar en la gestión de los procesos que conforman la cadena de suministro.

\section{METODOLOGİA}

El modelo de referencia SCOR se ha convertido en una guía cada vez más utilizada para encontrar mejoras en los procesos y aplicación de buenas prácticas en la cadena de suministro de cualquier sector productivo (Curbelo \& Delgado, 2014). Existen varias investigaciones que aplican este modelo para obtener resultados positivos en la administración de la cadena de suministros en sectores como el biodiesel, grasas, carnes, sistemas de riego, producción de leche, aprovechamiento de madera (Dissanayake \& Cross, 2018; Naranjo, Reyes \& Rodríguez, 2012; Salazar, Cavazos \& Martínez, 2012); sin embargo, en el sector de la generación abono orgánico no se ha aplicado y es la oportunidad encontrada en esta investigación.

De igual forma, se encuentran muy pocos referentes de aplicación del modelo SCOR en empresas pequeñas y en un contexto de países subdesarrollados. Por lo que se hace importante analizar la aplicación del modelo y su comportamiento a través de sus métricas en este contexto y aportar a la literatura nuevos hallazgos.

Este estudio se organiza en dos etapas, una relacionada con el diagnóstico de las 15 empresas analizadas y agremiadas en el departamento Norte de Santander (Colombia) con actividad productiva en la generación de abono orgánico a base de la lombriz roja californiana, utilizando el modelo SCOR. Como segunda etapa se plantea la estructuración de una SCORCARD con algunas métricas sugeridas por el modelo SCOR que se adaptan al contexto de producción y a los objetivos de la agremiación.

El procedimiento para la aplicación del modelo SCOR está basado en sus procesos principales (Supply Chain Council, 2012; Sanabria \& Bello, 2009):

1. Procesos de planeación: Desarrollo del Proyecto (Plan)

2. Procesos Fuente: Materias primas o insumos para el proceso productivo (Source)

3. Procesos de transformación: Obtención del abono orgánico a través de la lombriz californiana (Make)

4. Procesos de entrega: Distribución, transporte y comercialización (Delivery)

5. Procesos post-delivery: logística inversa, otros productos o subproductos (Return)

La información necesaria para realizar esta investigación se obtiene directamente desde la asociación de lombricultores, con el fin de tener información directa y confiable, sobre el manejo de la cadena de suministro actual y de las actividades que realizan en cada proceso. La recolección de 
información se hace a través de una entrevista, la cual se divide en dos momentos: una primera instancia con los gerentes de las empresas objeto de estudio para analizar procesos de planificación, $\mathrm{y}$ un segundo momento con los encargados de la producción para identificar información con respecto a los procesos fuente, transformación, distribución y devoluciones.

Partiendo de los indicadores que propone el modelo SCOR en cada conjunto de procesos, se hace la evaluación en la cadena de abastecimiento de la fabricación de abono orgánico a través de las secreciones de la lombriz californiana, recolectando la información para su medición. Posteriormente, se identifican los procesos que hacen parte de la Cadena de Suministro (Figura 1) para la fabricación del abono orgánico, la cual se encuentra estructurada en los siguientes eslabones a partir de subprocesos:

- Eslabón 1: Subproceso reproducción de las lombrices (Source) como insumo para el eslabón próximo.

- Eslabón 2: Mezcla de lombrices y fabricación del abono; en este subproceso se puede considerar un nuevo producto en logística inversa, obteniendo el humus purificado (transformación).

- Eslabón 3: Es el almacenamiento, despacho, transporte y entrega del abono en los diferentes puntos de distribución (deliver).

Con respecto al eslabón correspondiente a la Planificación, se encuentra en toda la cadena de suministro, desde la reproducción de las lombrices hasta la entrega en los puntos de distribución. Este proceso se retroalimenta constantemente teniendo en cuenta los indicadores de desempeño de la Tabla 1, que muestra la clasificación según los atributos que propone el modelo SCOR, los principales indicadores de primer eslabón teniendo en cuenta si su medición es de carácter interno o externo.

SCOR contiene tres niveles de detalle de procesos: Nivel Superior (Tipos de Procesos), Nivel de Configuración (Categorías de Procesos) y Nivel de Elementos de Procesos (Descomposición de los Procesos), en los tres niveles, SCOR aporta Indicadores Clave de desempeño (KPI). Estos Indicadores se dividen sistemáticamente en cinco Atributos de Rendimiento: Fiabilidad en el Cumplimiento, Flexibilidad, Velocidad de Atención, Coste y Activos (Lama \& Esteban, 2005).

Los Indicadores de Nivel 1 son medidas de alto nivel que recorren múltiples Procesos de SCOR y en la Tabla 1 se muestran para analizar si son de medición interna o externa teniendo en cuenta la información recolectada de las empresas. Seguidamente, en el nivel dos del modelo SCOR se tiene la aplicación de los procesos y la identificación de las métricas de medición acordes a cada subproceso para ser evaluados en el contexto actual de la cadena de abastecimiento de la producción de abono orgánico a través de la lombriz californiana.

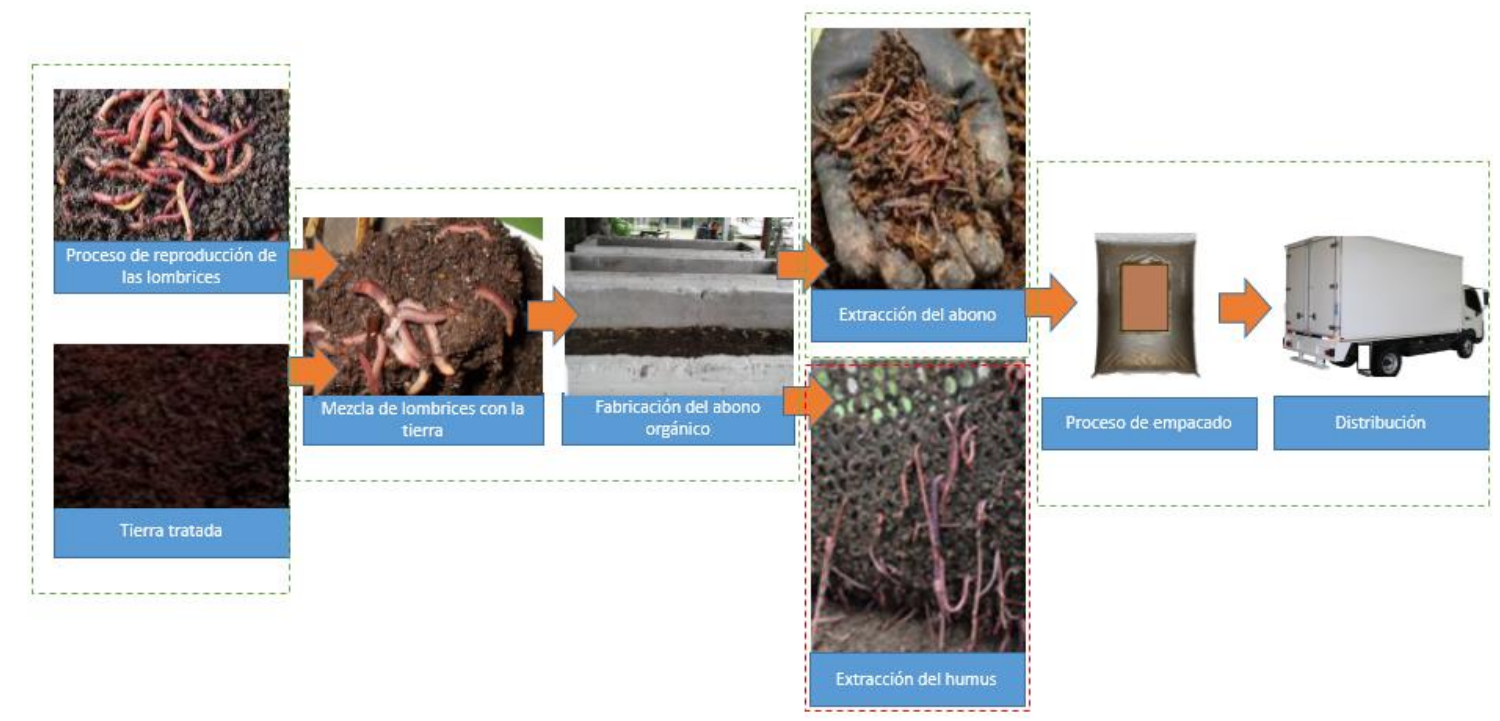

Figura 1. Cadena de Suministro de la producción de abono a través de la lombriz roja californiana (Elaboración propia, 2010). 


\section{RESULTADOS}

Teniendo en cuenta la información recolectada, la Tabla 3 muestra los resultados de la medición de los indicadores seleccionados (Tabla 2) de cada categoría del modelo SCOR, como diagnóstico del estado actual del funcionamiento de la cadena de suministro.

La Tabla 3 muestra el estado actual en promedio de las respuestas obtenidas en cada categoría del estado actual de las mediciones y de la meta que se propone cada una de las empresas. Se calcula la brecha entre lo actual y la meta para luego estimar la oportunidad de mejora.

Se evidencia que existe un grado de oportunidad para mejorar en cada una de las categorías evaluadas por empresa. Con la aplicación del modelo SCOR se puede estandarizar las métricas de medición de las cadenas de abono orgánico en las 15 empresas objeto de estudio y facilitar la evaluación a nivel gremial.
Se analiza que la medición realizada de los indicadores refleja que se están cumpliendo en su mayoría y evidencia en un primer diagnóstico que existe una armonía en los procesos de las cadenas analizadas (por empresa). Se deben replantear las metas en algunas de las empresas analizadas porque ya se están cumpliendo y se deben proponer otros frentes de trabajo que permita reflejar mejoras en la prestación del servicio al cliente.

La categoría confiabilidad de la cadena de suministro (Entregas de pedidos) muestra que si se puede generar una mejora contabilizada en un $40 \%$, ya sea replanteado los procesos de funcionamiento de la cadena o adaptando los indicadores. Partiendo del diagnóstico se evalúa la necesidad de seguir midiendo los indicadores iniciales y se revisan las métricas propuestas por el modelo SCOR en cada nivel para proponer una SCORCARD como instrumento importante para la administración de la cadena.

Tabla 3. Diagnóstico de rendimiento de la producción de abono orgánico a través de la lombriz californiana (Adaptado de SCOR)

\begin{tabular}{|c|c|c|c|c|c|}
\hline $\begin{array}{l}\text { Atributo o } \\
\text { categoría }\end{array}$ & Métricas de evaluación & Actual & Meta & Brecha & Oportunidad \\
\hline \multirow{3}{*}{$\begin{array}{l}\text { Confiabilidad de } \\
\text { la cadena de } \\
\text { suministro }\end{array}$} & Cumplimiento perfecto de ordenes & $69 \%$ & $90 \%$ & $21 \%$ & \multirow{3}{*}{$45 \%$} \\
\hline & No de ordenes entregadas completas & $75 \%$ & $90 \%$ & $15 \%$ & \\
\hline & Diferentes tipos del producto & 3 & 5 & 2 & \\
\hline \multirow{4}{*}{$\begin{array}{l}\text { Flexibilidad de } \\
\text { la cadena de } \\
\text { suministro }\end{array}$} & $\begin{array}{l}\text { Tiempo promedio del proceso de } \\
\text { compra de insumos (días) }\end{array}$ & 3 & 1 & -2 & \multirow{4}{*}{$\begin{array}{l}\text { Supera la meta } \\
\text { debe replantearse }\end{array}$} \\
\hline & $\begin{array}{l}\text { Tiempo promedio del proceso de } \\
\text { Fabricación del abono orgánico (días) }\end{array}$ & 24 & 20 & -4 & \\
\hline & $\begin{array}{l}\text { Tiempo promedio de riego para } \\
\text { mantener la humedad y temperatura de } \\
\text { las lombrices (minutos al día) }\end{array}$ & 30 & 20 & -10 & \\
\hline & $\begin{array}{l}\text { Tiempo promedio de empacado de la } \\
\text { producción (horas) }\end{array}$ & 12 & 8 & -4 & \\
\hline \multirow{5}{*}{$\begin{array}{l}\text { Agilidad en la } \\
\text { cadena de } \\
\text { suministro }\end{array}$} & $\begin{array}{l}\text { Días para incrementar el } \\
\text { aprovisionamiento en un } 20 \%\end{array}$ & 3 & 1 & -2 & \multirow{5}{*}{$\begin{array}{l}\text { Supera la meta } \\
\text { debe replantearse }\end{array}$} \\
\hline & $\begin{array}{l}\text { Días para incrementar la } \\
\text { producción de abono orgánico en un } \\
20 \%\end{array}$ & 1 & 1 & 0 & \\
\hline & $\begin{array}{l}\text { Días para incrementar la entrega } \\
\text { o distribución de acuerdo al } \\
\text { incremento de la producción }\end{array}$ & 1 & 1 & 0 & \\
\hline & Máx \% de incremento en producción & $1 \%$ & $20 \%$ & $-19 \%$ & \\
\hline & $\begin{array}{l}\text { Máximo \% de incremento en la } \\
\text { distribución y entrega }\end{array}$ & $1 \%$ & $20 \%$ & $-19 \%$ & \\
\hline \multirow{2}{*}{$\begin{array}{l}\text { Costos de la } \\
\text { Cadena de } \\
\text { Suministro }\end{array}$} & $\begin{array}{l}\text { Costo de mantener la cadena de } \\
\text { suministro }\end{array}$ & $100 \%$ & $88 \%$ & $-12 \%$ & \multirow{2}{*}{$\begin{array}{l}\text { Supera la meta } \\
\text { debe replantearse }\end{array}$} \\
\hline & Costo de planear & $90 \%$ & $75 \%$ & $-15 \%$ & \\
\hline
\end{tabular}




\begin{tabular}{|c|c|c|c|c|c|}
\hline $\begin{array}{c}\text { Atributo o } \\
\text { categoría }\end{array}$ & Métricas de evaluación & Actual & Meta & Brecha & Oportunidad \\
\hline & Costo de producir & $25 \%$ & $10 \%$ & $-15 \%$ & \\
\hline & Costo de entregar o distribuir & $10 \%$ & $8 \%$ & $-2 \%$ & \\
\hline & Costo de almacenamiento transitorio & $12 \%$ & $7 \%$ & -5 & \\
\hline \multirow{2}{*}{$\begin{array}{c}\text { Manejo de } \\
\text { Activos en la } \\
\text { Cadena de } \\
\text { Suministro }\end{array}$} & $\begin{array}{l}\text { Ciclo de flujo de efectivo para } \\
\text { Abono Orgánico (días) }\end{array}$ & 5 & 3 & -2 & \multirow{2}{*}{$\begin{array}{l}\text { Supera la meta } \\
\text { debe replantearse }\end{array}$} \\
\hline & $\begin{array}{l}\text { Ciclo de flujo de efectivo para } \\
\text { Proveedores (días) }\end{array}$ & 30 & 30 & 0 & \\
\hline
\end{tabular}

Tabla 4. Indicadores propuestos para la medición del desempeño de la cadena de abastecimiento de la producción de abono orgánico por medio de la lombriz roja californiana (Adaptado de SCOR versión 2018)

\begin{tabular}{|c|c|c|}
\hline Categoría & $\begin{array}{c}\text { Código } \\
\text { modelo SCOR }\end{array}$ & Indicador \\
\hline \multirow{4}{*}{ Fiabilidad } & RL.1.1 & Cumplimiento perfecto de ordenes \\
\hline & RL.2.1 & Número de ordenes entregadas completas \\
\hline & RL.2.4 & Diferentes presentaciones del producto \\
\hline & RL.2.3 & $\begin{array}{l}\text { Precisión y diligenciamiento de la documentación } \\
\text { de entrega de pedidos }\end{array}$ \\
\hline \multirow{3}{*}{ Sensibilidad } & RS.2.1 & $\begin{array}{l}\text { Tiempo promedio del proceso de } \\
\text { compra de insumos (días) }\end{array}$ \\
\hline & RS.2.2 & $\begin{array}{l}\text { Tiempo promedio del proceso de } \\
\text { Fabricación del abono orgánico (días) }\end{array}$ \\
\hline & RS.1.1 & Tiempo promedio de entrega de pedidos (días) \\
\hline \multirow{5}{*}{$\begin{array}{l}\text { Costos de la } \\
\text { Cadena de Suministro }\end{array}$} & C.O.1.1 & Costo total de gestión de la cadena de suministro \\
\hline & C.0.2.3 & Costo de fabricación \\
\hline & C.0.2.5 & Costo de devoluciones de pedidos \\
\hline & C.O.2.8 & Costo directo de materiales \\
\hline & C.0.2.7 & Costo directo de mano de obra \\
\hline $\begin{array}{l}\text { Manejo de Activos en la } \\
\text { Cadena de Suministro }\end{array}$ & A.M.1.1 & $\begin{array}{l}\text { Ciclo de flujo de efectivo para } \\
\text { Abono Orgánico (días) }\end{array}$ \\
\hline \multirow{4}{*}{$\begin{array}{l}\text { Agilidad en la cadena de } \\
\text { suministro }\end{array}$} & A.M.1.3 & Retorno sobre capital de trabajo \\
\hline & A.M.2.4 & Ingresos de la cadena de suministro \\
\hline & A.G.1.1 & Adaptabilidad de la cadena de suministro \\
\hline & A.G.1.4 & Valor general de los riesgos \\
\hline
\end{tabular}

La Tabla 4 muestra los indicadores propuestos, además de los anteriormente medidos replanteando las metas, para la medición del desempeño de la cadena de abastecimiento de la producción de abono orgánico por medio de la lombriz roja californiana. Las estrategias que se planteen a nivel gerencial para el mejoramiento de la producción de abono orgánico a base de la lombriz roja californiana deben estar acorde con los indicadores propuestos.

\section{CONCLUSIONES}

En el presente trabajo se evidenció que el modelo SCOR puede ser una herramienta muy útil a la hora de educar a la mano de obra participante de la fabricación de abono orgánico en la importancia de buscar constantemente mejoras del proceso y reducción de costos en los eslabones de la cadena de suministro. El productor a lo largo del proyecto mostro gran interés en la posibilidad de bajar costos en la producción y demás aspectos de la cadena de suministro que se identificaba durante el estudio, manifestaban la importancia de reinvertir 
en mejorar la calidad del producto y los requisitos para la exportación del mismo. La Figura 2 muestra la cadena identificada en ejercicio con los productores de forma estandarizada para las 15 empresas participantes del estudio.

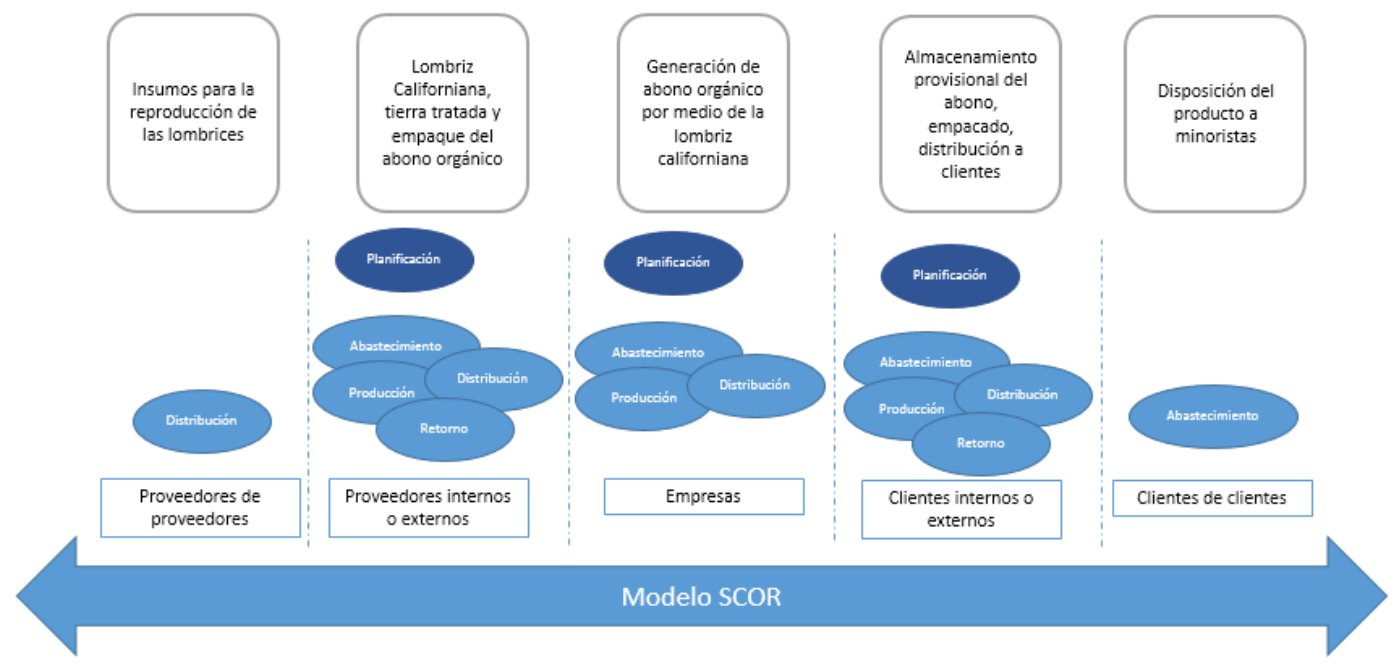

Figura 2. Cadena de Abastecimiento en la Producción de Abono Orgánico (Adaptado modelo SCOR)

En el desarrollo de esta investigación se deja ver que las empresas productoras de abono orgánico de la muestra estudiada no conocen sobre la formalidad de las cadenas de abastecimiento y mucho menos de las metodologías como SCOR que permiten hacer una evaluación constante de la producción con miras a mejorar continuamente. Los empresarios hacen la producción de acuerdo con pronósticos a priori y según experiencias de años, pero sin una planificación de estas.

La administración de la cadena de abastecimiento es un aspecto poco visto en el sector de investigación, manifiestan no tener el personal que planifique la producción ni los conocimientos para hacerlo, debido a que en su mayoría son propietarios de empresas sin niveles de estudio o capacitación adecuada. Muy pocas veces los estudios analizan toda una cadena de abastecimiento por sus diversos factores influyentes. Es importante que se realice un análisis de la cadena completa y no por pequeños procesos porque genera mejores resultados, permite evidenciar aspectos importantes de comunicación interna y externa, mejorar alianzas bajo una visión holística.

El modelo SCOR permite que se evidencien nuevos negocios u oportunidades con valores agregados en el mercado, que sin duda alguna permiten mayor competitividad de las empresas. Además, dentro de la cadena de suministro de las empresas objeto de estudio, productoras de abono orgánico a través de la lombriz californiana, es importante en el proceso desde la recolección de la materia prima hasta la elaboración del producto final.

De este modo los, factores clave como los resultados comerciales, calidad de productos y la satisfacción del cliente son el centro para la implementación de mejores prácticas, la búsqueda del mejoramiento continuo a través de la evaluación constante (Salazar, Cavazos, \& Martínez, 2012). Esta investigación deja abierta la posibilidad para evaluar las capacidades gerenciales de los dueños de empresas del sector productivo y el mejoramiento de procesos a través de métodos que permitan la reducción de costos, mejoramiento de la competitividad y puesta en marcha de nuevas oportunidades de mercado.

\section{REFERENCIAS}

Akkawuttiwanich, P., \& Yenradee, P. (2018). Fuzzy QFD enfoque para gestionar los indicadores de rendimiento SCOR. Informática e Ingeniería Industrial , 189201.

Council, S. C. (2012). Supply Chain Operations Reference Model. United States of America: APIC.

Curbelo, A. D., \& Delgado, F. M. (2014). EL MODELO SCOR Y EL BALANCED SCORECARD, UNA PODEROSA COMBINACIÓN INTANGIBLE PARA 
LA GESTION EMPRESARIAL. Revista Científica Visión de Futuro, 36-57.

Curbelo, A. D., \& Delgado, F. M. (2014). El modelo SCOR y el Balanced Scorecard: una poderosa combinación intangible para la gestión empresarial. Visión de Futuro, 36-57.

Curbelo, A. D., \& Delgado, F. M. (2014). El modelo SCOR y el Balanced Scorecard: una poderosa combinación intangible para la gestión empresarial. Vision de futuro, 36-57.

Delipinar, G. E., \& Kocaoglu, B. (2016). Using SCOR model to gain competitive advantage: A Literature review. Procedia - Social and Behavioral Sciences, 398406.

Dissanayake, C. K., \& A.Cross, J. (2018). Systematic mechanism for identifying the relative impact of supply chain performance areas on the overall supply chain performance using SCOR model and SEM. International Journal of Production Economics, 102-115.

Lama, J. L., \& Esteban, F. C. (2005). Análisis del modelo SCOR para la Gestión de la Cadena de Suministro. IX Congreso de Ingeniería de Organización , 1-10.

Lepori, E., Damand, D., \& Barth, B. (2013). Benefits and limitations of the SCOR model in warehousing. Procesos de la IFAC Volúmenes, 424-429.

Mazo, A. Z., Montoya, R. A., \& Henao, S. A. (2014). Indicadores logísticos en la cadena de suministro como apoyo al modelo scor. Revista Clío América, 90 110.

Mendoza, J. A. (2014). Analytical hierarchy process and SCOR model to support supply chain re-design. International Journal of Information Management, 634638.

Naranjo, J. I., Reyes, C. M., \& Rodríguez, J. C. (2012). Diagnóstico basado en el Modelo Scor para la cadena de suministro de la empresa Matecsa S.A. AVANCES Investigación en Ingeniería , 94-101.

Naranjo, J. I., Reyes, C. M., \& Rodríguez, J. C. (2012). Diagnóstico basado en el Modelo Scor para la cadena de suministro de la empresa Matecsa S.A. AVANCES Investigación en Ingeniería, 94-101.

Penagos, C. O., \& Rodríguez, J. M. (2009). Situación actual de la comercialización del abono orgánico Bocashi en el Sugamuxi. Cuadernos de administración, 141-154.

Salazar, F., Cavazos, J., \& Martínez, J. L. (2012). Metodología basada en el Modelo de Referencia para Cadenas de Suministro para Analizar el Proceso de producción de Biodiesel a partir de Higuerilla. Información Tecnológica, 47-56.

Sanabria, H. F., \& Bello, C. A. (2009). Propuesta metodológica para la aplicación del modelo Supply Chain Operations Reference. Ciencia Investigación Academia Desarrollo, 34-41.

Santiago, F. A. (2006). La Gestión de Cadenas de Suministros: Un enfoque de integración global de procesos. Visión Gerencial, 5362.

Santos, Y. L. (2019). La administración de la cadena de suministro sustentable y las pequeñas y medianas empresas de economías emergentes: caso México. Revista Iberoamericana de Contaduría, Economía y Administración , 1-28.

Souza, B. d., Carmo, D. L., Santos, R. H., \& Fernandes, R. B. (2018). Perceptions of agroecological farmers on green manure use in Southeast Minas Gerais, Brazil. . Idesia (Arica), 15-25.

Supply Chain Council. (2008). SCOR Overview version 9.0. United States: Supply Chain Council.

Supply Chain Council. (2012). Supply Chain Operations Reference Model. United States of America: APIC. 
SCOR 11.0

ANEXOS

\begin{tabular}{|c|c|c|}
\hline ID SCOR & DESCRIPCIÓN & PREGUNTAS \\
\hline SP1 & $\begin{array}{l}\text { Planeación de la cadena de } \\
\text { suministros }\end{array}$ & $\begin{array}{l}\text { 1. En los dos últimos años cuál ha sido el costo de } \\
\text { funcionamiento de la cadena de suministro? } \\
\text { 2. Se cumple con la meta propuesta de costos? } \\
\text { 3. Considera que el costo de planeación que se asume } \\
\text { para la operación de la cadena de suministro está } \\
\text { acorde con las mestas propuestas? } \\
\text { 4. Considera que el costo de entregar o distribuir que } \\
\text { se asume está acorde con las mestas propuestas? } \\
\text { 5. Los costos de almacenamiento transitorio de materia } \\
\text { prima y producto termino están acorde con las metas } \\
\text { propuestas? } \\
\text { 6. Cómo es el comportamiento del flujo de efectivo } \\
\text { por producto terminado en la gestión de la cadena? } \\
\text { 7. Cómo es el comportamiento del flujo de efectivo de } \\
\text { proveedores en la gestión de la cadena? }\end{array}$ \\
\hline SP2 & Planeación de los insumos & $\begin{array}{l}\text { 8. Cuál es el promedio en días para la obtención de } \\
\text { materia prima? } \\
\text { 9. Si se quisiera incrementar en } 20 \% \text { la producción, } \\
\text { según la meta de la asociación, cuántos días se } \\
\text { requieren para incrementar el aprovisionamiento? }\end{array}$ \\
\hline $\begin{array}{l}\text { SP3 } \\
\text { SSR3 }\end{array}$ & Planeación de la fabricación & $\begin{array}{l}\text { 10. Existe variedad en la presentación de la entrega del } \\
\text { producto terminado? } \\
\text { 11. Cuál es el tiempo promedio del proceso de } \\
\text { fabricación del abono orgánico (días)? Cree que se } \\
\text { puede mejorar? } \\
\text { 12. Cuál es el tiempo promedio de riego para mantener } \\
\text { la humedad y temperatura de las lombrices (minutos al } \\
\text { día)? } \\
\text { 13. Cuál es el tratamiento del exceso de producto? }\end{array}$ \\
\hline SP4 & Planeación de la entrega & $\begin{array}{l}\text { 14. Actualmente, se cumple perfectamente con las } \\
\text { especificaciones de las órdenes de compra? } \\
\text { 15. El número de ordenes entregadas completas está } \\
\text { acorde con la meta propuesta? }\end{array}$ \\
\hline SP5 & $\begin{array}{l}\text { Planeación de las } \\
\text { devoluciones }\end{array}$ & $\begin{array}{l}\text { 16. Cuáles son los principales motivos de devolución } \\
\text { de un producto? } \\
\text { 17. Cuántas veces en el año reciben devoluciones? }\end{array}$ \\
\hline $\mathrm{SS} 2-\mathrm{SM} 2$ & Fabricación por pedidos & $\begin{array}{l}\text { 18. Si se incrementar la producción en } 20 \% \text { como está } \\
\text { estipulado en la meta de la asociación, cuántos días se } \\
\text { requieren para incrementar la entrega o distribución de } \\
\text { la producción? }\end{array}$ \\
\hline SS3 & $\begin{array}{l}\text { Fabricación por solicitud de } \\
\text { ingeniería }\end{array}$ & $\begin{array}{l}\text { 19. La cadena está preparada para responder a la } \\
\text { solicitud eventual de un pedido no programado? }\end{array}$ \\
\hline SS1 & $\begin{array}{l}\text { Control de producto } \\
\text { almacenado }\end{array}$ & $\begin{array}{l}\text { 20. Cuál es el tiempo promedio de empacado de la } \\
\text { producción (horas)? }\end{array}$ \\
\hline
\end{tabular}




\begin{tabular}{|l|l|l|}
\hline ID SCOR & \multicolumn{1}{|c|}{ DESCRIPCIÓN } & \multicolumn{1}{c|}{ PREGUNTAS } \\
\hline SE3 & $\begin{array}{l}\text { Gestión de datos e } \\
\text { información }\end{array}$ & $\begin{array}{l}\text { 21. Existe control de la documentación de las } \\
\text { entregas? } \\
\text { 22. Utilizan algún sistema computacional para el } \\
\text { control de los pedidos, entregas y distribución? }\end{array}$ \\
\hline SE1 & $\begin{array}{l}\text { Gestión de las reglas de } \\
\text { negocio de la cadena de } \\
\text { suministro }\end{array}$ & $\begin{array}{l}\text { 23. Cómo es su relación con los socios? } \\
\text { 24. Cómo es su relación con proveedores? } \\
\text { 25. Existen más de dos proveedores de materia prima? } \\
\text { garanticen la venta de la producción? } \\
\text { 27. A qué países se exporta el producto terminado y } \\
\text { los subproductos del proceso de fabricación? }\end{array}$ \\
\hline SE2 & Gestión del rendimiento & $\begin{array}{l}\text { 28. Cuál puede ser el máximo \% de incremento en la } \\
\text { producción según capacidad instalada? } \\
\text { 29. Cuál puede ser el máximo \% de incremento en la } \\
\text { distribución y entrega según capacidad instalada? }\end{array}$ \\
\hline SE8 & Cumplimiento de normas & $\begin{array}{l}\text { 30. Actualmente la producción de abono orgánico } \\
\text { cumple con las normas sanitarias? } \\
\text { 31. La empresa cuenta con alguna certificación de la } \\
\text { producción? }\end{array}$ \\
\hline SE7 & Gestión de redes de trabajo & $\begin{array}{l}\text { 32. Cómo es el proceso de la consecución de nuevos } \\
\text { clientes? } \\
\text { 33. Con cuales actores tiene mayor contacto para el } \\
\text { desarrollo del negocio? (universidades, proveedores, } \\
\text { entidades del estado, otras) } \\
\text { 34. La asociación apoya constantemente el } \\
\text { funcionamiento de la empresa? }\end{array}$ \\
\hline & & \\
& &
\end{tabular}

